Article

\title{
Advantages of 18F-FDG PET/CT Imaging over Modified Duke Criteria and Clinical Presumption in Patients with Challenging Suspicion of Infective Endocarditis
}

\author{
Valentin Pretet ${ }^{1}$, Cyrille Blondet ${ }^{1,2}$, Yvon Ruch 2,3 ${ }^{(D}$, Matias Martinez 1,4,5, Soraya El Ghannudi ${ }^{1,6}$, \\ Olivier Morel ${ }^{7}$, Yves Hansmann ${ }^{2,3}$, Thomas H. Schindler ${ }^{8}$ and Alessio Imperiale ${ }^{1,2,9, *}$
}

Citation: Pretet, V.; Blondet, C.; Ruch, Y.; Martinez, M.; El Ghannudi, S.; Morel, O.; Hansmann, Y.; Schindler, T.H.; Imperiale, A. Advantages of 18F-FDG PET/CT Imaging over Modified Duke Criteria and Clinical Presumption in Patients with Challenging Suspicion of Infective Endocarditis. Diagnostics 2021, 11, 720. https://doi.org/ 10.3390 /diagnostics11040720

Academic Editor: Giorgio Treglia

Received: 29 March 2021

Accepted: 16 April 2021

Published: 18 April 2021

Publisher's Note: MDPI stays neutral with regard to jurisdictional claims in published maps and institutional affiliations.

Copyright: (c) 2021 by the authors. Licensee MDPI, Basel, Switzerland. This article is an open access article distributed under the terms and conditions of the Creative Commons Attribution (CC BY) license (https:// creativecommons.org/licenses/by/ $4.0 /)$.
1 Nuclear Medicine and Molecular Imaging, ICANS, University Hospitals of Strasbourg, rue Albert Calmette, 67093 Strasbourg, France; valentin.pretet@hotmail.com (V.P.); c.blondet@icans.eu (C.B.); matias.martinezag@hotmail.com (M.M.); soraya.elghannudi-abdo@chru-strasbourg.fr (S.E.G.)

2 Faculty of Medicine, FMTS, University of Strasbourg, 67000 Strasbourg, France; yvon.ruch@chru-strasbourg.fr (Y.R.); yves.hansmann@chru-strasbourg.fr (Y.H.)

3 Infectious Diseases, University Hospitals of Strasbourg, 67000 Strasbourg, France

Nuclear Medicine and Molecular Imaging, Oulton Institute, 5000 Cordoba, Argentina

Nuclear Medicine, Hospital Privado Universitario, 5000 Cordoba, Argentina

Radiology, University Hospitals of Strasbourg, 67000 Strasbourg, France

Cardiology, University Hospitals of Strasbourg, 67000 Strasbourg, France; olivier.morel@chru-strasbourg.fr

Mallinckrodt Institute of Radiology, Division of Nuclear Medicine, Washington University,

St Louis, MO 63110, USA; thscindler@wustl.edu

9 Molecular Imaging-DRHIM, IPHC, UMR 7178, CNRS, 67037 Strasbourg, France

* Correspondence: a.imperiale@icans.eu; Tel.: +33-368-767-448; Fax: +33-368-767-256

\begin{abstract}
According to European Society of Cardiology guidelines (ESC2015) for infective endocarditis (IE) management, modified Duke criteria (mDC) are implemented with a degree of clinical suspicion degree, leading to grades such as "possible" or "rejected" IE despite a persisting high level of clinical suspicion. Herein, we evaluate the ${ }^{18}$ F-FDG PET/CT diagnostic and therapeutic impact in IE suspicion, with emphasis on possible/rejected IE with a high clinical suspicion. Excluding cases of definite IE diagnosis, 53 patients who underwent ${ }^{18} \mathrm{~F}-\mathrm{FDG}$ PET/CT for IE suspicion were selected and afterwards classified according to both $\mathrm{mDC}$ (possible IE/Duke 1, rejected IE/Duke 0) and clinical suspicion degree (high and low IE suspicion). The final status regarding IE diagnosis (gold standard) was based on the multidisciplinary decision of the Endocarditis Team, including the 'imaging specialist'. PET/CT images of the cardiac area were qualitatively interpreted and the intensity of each focus of extra-physiologic ${ }^{18}$ F-FDG uptake was evaluated by a maximum standardized uptake value (SUVmax) measurement. Extra-cardiac ${ }^{18}$ F-FDG PET/CT pathological findings were considered to be a possible embolic event, a possible source of IE, or even a concomitant infection. Based on the Endocarditis Team consensus, final diagnosis of IE was retained in 19 (36\%) patients and excluded in $34(64 \%)$. With a sensitivity, specificity, positive predictive value (PPV), negative predictive value (NPV), and global accuracy of 79\%,100\%, 100\%,89\%, and 92\%, respectively, PET/CT performed significantly better than $\operatorname{mDC}(p=0.003)$, clinical suspicion degree $(p=0.001)$, and a combination of both $(p=0.001)$ for IE diagnosis. In 41 patients with possible/rejected IE but high clinical suspicion, sensitivity, specificity, PPV, NPV, and global accuracies were $78 \%, 100 \%, 100 \%, 85 \%$, and $90 \%$, respectively. Moreover, PET/CT contributed to patient management in 24 out of 53 (45\%) cases. ${ }^{18}$ F-FDG PET/CT represents a valuable diagnostic tool that could be proposed for challenging IE cases with significant differences between $\mathrm{mDC}$ and clinical suspicion degree. ${ }^{18} \mathrm{~F}$-FDG PET/CT allows a binary diagnosis (definite or rejected IE) by removing uncertain diagnostic situations, thus improving patient therapeutic management.
\end{abstract}

Keywords: infective endocarditis; modified Duke criteria; 18F-FDG PET/CT 


\section{Introduction}

Infective endocarditis (IE) is an uncommon and challenging infectious disease associated with severe complications and high mortality despite major advances in diagnostic and therapeutic strategies [1,2]. In the last few decades, we have witnessed a continuous evolution of the diagnostic criteria used in clinical practices for the management of patients with suspected IE. In 2000, modified Duke criteria (mDC), including clinical, echocardiographic, and biological findings, as well as blood cultures and serological results, were recommended for diagnostic use [3,4]. However, $\mathrm{mDC}$ show inadequate accuracy for IE diagnosis, particularly in patients with prosthetic valve endocarditis (PVE) and pacemaker or defibrillator lead IE, for which echocardiography is inconclusive in up to one-third of patients [5]. This is particularly true in early stages of the infectious process when the physician could have high clinical suspicion without a corresponding adequate $\mathrm{mDC}$ score for making the IE diagnosis. Consequently, the concept of clinical probability of IE (i.e., presumption of infection) has been merged with $\mathrm{mDC}$ in the recent diagnostic recommendations of the European Society of Cardiology (ESC) [4]. Hence, at present, diagnosis of IE is usually based on clinical probability in combination with $\mathrm{mDC}$ (definite, possible, or rejected IE), which are dependent on the diagnostic accuracy of either transthoracic (TTE) or transesophageal echocardiography (TEE). It is therefore possible to grade patients as having "possible" or even "rejected" IE despite a persisting high level of clinical suspicion [3,4].

As highlighted in 2015 by the revised guidelines of the European Society of Cardiology (ESC) [4], imaging is becoming increasingly significant for the management of patients with suspected IE [6]. Indeed, new techniques are becoming more frequently used when TTE/TEE results are negative or doubtful in both "possible" and "rejected" diagnoses, with a persistent high level of clinical suspicion. 18F-fluorodesoxyglucose (18F-FDG) positron emission tomography/computed tomography (PET/CT) emerged as a critical tool for identification of infected cardiovascular device implantations in cases where echocardiographic findings remain equivocal or negative despite a high clinical suspicion $[7,8]$. In addition, whole body 18F-FDG PET/CT potentially affords the identification of extra-cardiac primary infection sources and the extent of spread of the infection (infective embolism) [9].

Although the addition of a cardiac imaging exploration, be it MRI, CT, or PET/CT, is generally not recommended by ESC2015 guidelines, the interest of 18F-FDG PET/CT in patients with Duke 2 diagnosis (definite IE) has been shown by Duval et al. [10] in a recent prospective multicentric study, including 140 patients with IE suspicion and prosthetic (PV) or native valves (NV). When systematically performed, 18F-FDG PET/CT modified care in $31 \%$ of the 80 Duke 2 patients included in the study. Interestingly, Philip et al. [11] recently highlighted the central role of 18F-FDG PET/CT imaging in ESC2015 guidelines, resulting in correct patient reclassification from rejected or possible IE into definite IE. In their study, authors reported a 18F-FDG PET/CT sensitivity of $83.5 \%$, including 53 out of 115 cases of definite IE.

To this day, few studies have specifically focused on patients presenting with discordance between $\mathrm{mDC}$ and clinical presumption of ongoing infection $[7,8]$. In view of the above, the aim of the present retrospective single-center study was to evaluate the diagnostic and therapeutic impact of 18F-FDG PET/CT in the management of challenging cases of IE suspicion, with emphasis on patients with possible/rejected IE but with high clinical suspicion.

\section{Materials and Methods}

\subsection{Patient Population}

This is a non-interventional, monocentric, retrospective study involving patients addressed to the Nuclear Medicine Department of the Strasbourg University Hospitals (France) for ${ }^{18}$ F-FDG PET/CT between May 2013 to November 2019 for suspected IE concerning the native valve or prosthetic material in the cardiac area (i.e., prosthetic valve, pacemaker lead, intra cardiac left ventricular assist device (LVAD), or other devices). 
Patients with a definite IE diagnosis, according to $\mathrm{mDC}$, and patients with suspected infection developed on extra cardiac material (i.e., prosthetic vascular devices, pacemaker boxes, extra cardiac LVAD, and other devices) were not included in the analysis.

In the present study, each patient with IE suspicion has been classified twice, according to both $\mathrm{mDC}$ and clinical suspicion degree. Patients were initially categorized as possible IE (Duke 1) or rejected IE (Duke 0) and afterwards graded based on the clinical suspicion of infection (high or low suspicion of IE) (Figure 1). The clinical suspicion of endocarditis was defined by an infectious disease specialist based on available clinical, laboratory, and echocardiogram data. This was a subjective clinical judgment. Finally, patients were categorized considering both mDC (Duke 1 or 0 ) and clinical suspicion of IE (high or low suspicion). This approach was carried out blinded to the results of ${ }^{18} \mathrm{~F}-\mathrm{FDG}$ PET/CT. This methodology is based on ESC2015 recommendations, which combine mDC (Duke $0=$ rejected, 1 = possible, 2 = definite) and clinical suspicion of IE (low vs. high suspicion) in order to classify patients into three groups: definite IE, possible/rejected IE but high suspicion, and rejected IE with low suspicion. Indeed, even if the diagnostic probability of IE is rejected by $\mathrm{mDC}$, the clinical presumption can be strong enough (i.e., high suspicion degree) to consider a rejected IE as a possible IE [4], justifying further imaging examinations.

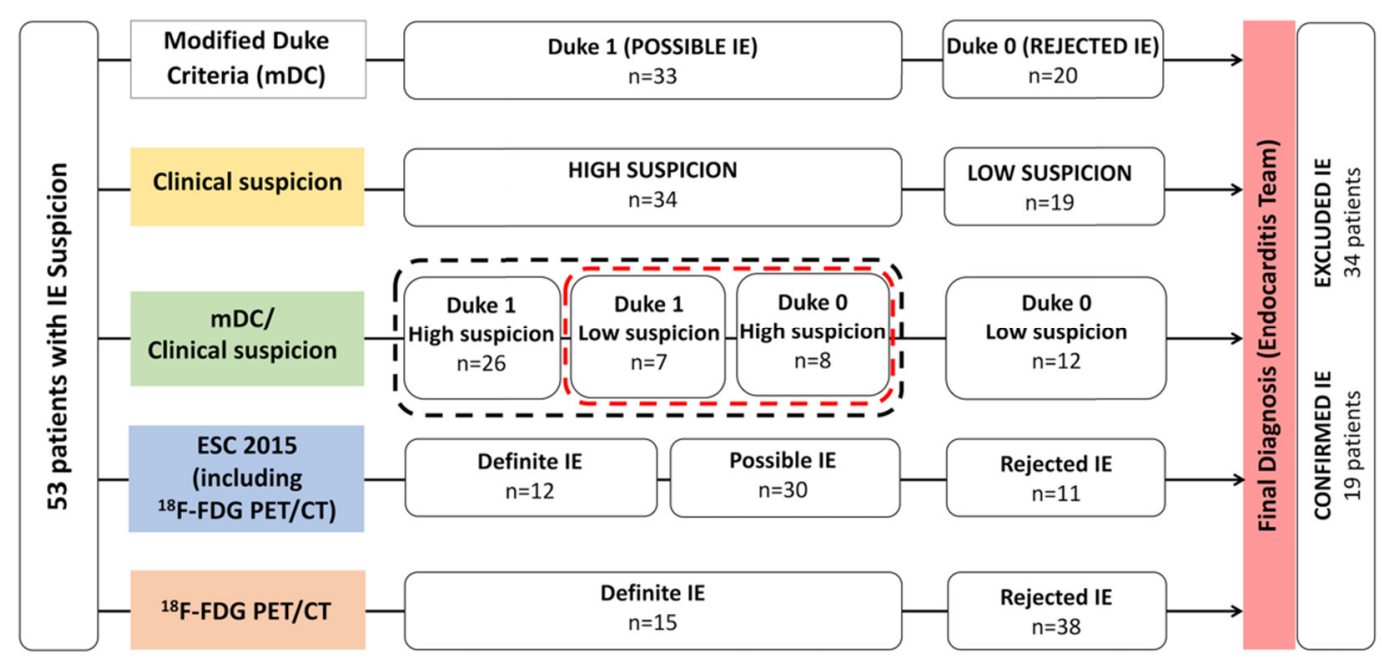

Figure 1. Main study design and patient population classifications. Black dotted line regroups patients needing cardiac imaging (Cardiac-CT, 18F-FDG PET/CT, or Cardiac-MRI) according to ESC 2015 guidelines. Red dotted line regroups the strongest classification discordances.

\section{2. ${ }^{18}$ F-FDG PET/CT: Technical Features and Interpretation Criteria}

All ${ }^{18} \mathrm{~F}$-FDG PET/CT were performed using an EARL-accredited combined PET/CT device (GE Healthcare, Chicago, IL, USA until April 2014, then Biograph mCT TOF, Siemens, Berlin, Germany) following the guidelines of the European Association of Nuclear Medicine (EANM). No patient with a previous history of thoracic surgery underwent ${ }^{18} \mathrm{~F}$ FDG PET/CT in the first three postoperative months. PET/CT acquisitions started about $60 \mathrm{~min}$ after 3-5 MBq/ $\mathrm{kg}$ of ${ }^{18} \mathrm{~F}-\mathrm{FDG}$ injection (Flucis, IBA, Paris, France), including a head to midthigh non-contrast enhanced CT (128 detectors-row, $140 \mathrm{kV}, 115 \mathrm{~mA}, 1$ s per rotation, pitch 0.8 , slice thickness of $1 \mathrm{~mm}$ ), followed by a PET scan (3-5 $\mathrm{min} /$ field). In order to minimize physiological myocardial ${ }^{18}$ F-FDG uptake, a high fat and low carbohydrate diet $24 \mathrm{~h}$ before the examination followed by $12 \mathrm{~h}$ of fasting was observed. Twenty-seven patients also received 10-20 UI/ $\mathrm{kg}$ standard heparin intravenous administration about 30 min before ${ }^{18}$ F-FDG injection. ECG-gated acquisition or contrast-enhanced PET/CTA was not performed in any case. PET data were reconstructed with and without CT-based attenuation correction using the common iterative algorithm OSEM. CT, PET, and combined PET/CT images were displayed on a dedicated workstation (Syngo.via VB30, Siemens, 
Berlin, Germany) and analyzed by two experienced nuclear medicine physicians. In case of disagreement, a third nuclear physician was required to reach consensus.

Attenuation-corrected and non-attenuation-corrected PET/CT images of the cardiac area were qualitatively interpreted according to widely accepted criteria (pronounced focal and/or heterogenous vs. absence of or homogenous mild ${ }^{18}$ F-FDG uptake around the device) $[12,13]$. Although in clinical practice the use of standardized uptake value (SUV) is debated and not always recommended [14,15], we have assessed SUVmax of each focus of extra-physiologic uptake of ${ }^{18} \mathrm{~F}-\mathrm{FDG}$ in cardiac area. The patient's clinical situation and previous history were considered for the assessment and interpretation of increased extraphysiologic uptake of ${ }^{18} \mathrm{~F}$-FDG. Extra-cardiac ${ }^{18} \mathrm{~F}$-FDG PET/CT pathological findings were considered to be a possible embolic event, a possible source of IE, or even a concomitant infection. ${ }^{18}$ F-FDG was used in the setting of an approved marketing authorization and a cross-disciplinary team, including clinicians and nuclear medicine physicians who stated about ${ }^{18} \mathrm{~F}$-FDG PET/CT indications. In accordance with local institutional guidelines, all patients included gave free and informed consent for the use of anonymous personal medical data extracted from their file for scientific or epidemiological purposes.

\subsection{Statistical Analysis}

Results for continuous data were expressed as mean \pm standard deviation or median and range as appropriate. Categorical variables were presented as numbers and percentages. The Wilcoxon-Mann-Whitney $U$ test and the McNemar test were used for comparison. ${ }^{18} \mathrm{~F}$-FDG PET/CT sensitivity (Se), specificity (Sp), positive predictive value (PPV), negative predictive value (NPV), and global accuracy (Ac) were calculated for each patient. The final status regarding IE diagnosis (gold standard) was based on the multidisciplinary decision of the Endocarditis Team, including the 'imaging specialist' [16]. Two-sided $p$ values $<0.05$ were considered significant. Statistical analyses were performed using an open access statistical software (biostatgv.sentiweb.fr, 2020).

\section{Results}

\subsection{Overall Population}

Fifty-three patients with suspected IE were enrolled during the study period. Table 1 summarizes the characteristics of patient population. Mean age was $65 \pm 19$ years with a male to female ratio of 3:2. In total, 43 out of $53(81 \%)$ patients had a cardiac device, $22(41 \%)$ a prosthetic valve, $24(45 \%)$ a cardiac electronic implantable device (CEID), 4 $(8 \%)$ a LVAD, and other devices were in the last 4 cases. Five $(9 \%)$ of the patients had at least two prosthetic valves and nine (17\%) had at least one prosthetic valve and a CEID (Table 1). Blood culture revealed causative pathogen in 24 out of 53 included patients (45\%). In the overall population, TTE and TEE were positive in $6(12 \%)$ and $13(30 \%)$ patients, respectively. According to $\mathrm{mDC}$ criteria, 20 (38\%) patients were classified as Duke 0 and 33 $(62 \%)$ as Duke 1. According to the Infectious Disease Specialist Consensus, $34(64 \%)$ and 19 $(36 \%)$ patients were classified with having low or high clinical suspicion of IE, respectively. Merging both mDC and clinical suspicion, 26 (49\%) patients were classified as having Duke $1 /$ high suspicion, 7 (13\%) patients as having Duke 1 /low suspicion, $8(15 \%)$ patients as having Duke 0/high suspicion, and 12 (23\%) patients as having Duke 0/low suspicion (Figure 1). Finally, based on the Endocarditis Team decision [16], the final diagnosis of IE was retained in $19(36 \%)$ patients and excluded in the remaining $34(64 \%)$. IE occurred on the prosthetic mitral valve in $2(11 \%)$ cases, on the prosthetic aortic valve in $3(16 \%)$, on the prosthetic pulmonary valve in $2(11 \%)$, on the native mitral valve in $2(11 \%)$, on the CEID intra cardiac lead in $6(32 \%)$, on the surgical patch closure of the atrial septum defect in 1 $(5 \%)$, and on the LVAD in $3(16 \%)$. Blood culture were conclusive in 12 out of 19 cases $(63 \%)$ with final diagnosis of EI and in 12 of 34 patients (35\%) without final diagnosis of EI. The main isolated pathogens responsible for infection in patients with EI final diagnosis were Staphylococcus aureus $(n=4)$, Coagulase Negative Staphylococcus $(n=3)$, Enterococcus 
spp. $(n=3)$, and Streptococcus spp. $(n=2)$. In 19 patients with final diagnosis of EI, TTE and TEE were positive in $3(16 \%)$ and $4(24 \%)$ cases, respectively.

Table 1. Population main characteristics.

\begin{tabular}{|c|c|}
\hline Characteristics & Values \\
\hline Age (years), mean $\pm \mathrm{SD}$ & $65 \pm 19$ \\
\hline \multicolumn{2}{|l|}{ Sex, $n(\%)$} \\
\hline Female & $20(38)$ \\
\hline Male & $33(62)$ \\
\hline CRP (mg/L), mean (range) & $81.9(4.0-280 ; \mathrm{N}<4)$ \\
\hline White blood cell (G/L), mean (range) & $10.0(2.8-20.0 ; 4.1<\mathrm{N}<10.5)$ \\
\hline Material, $n(\%)$ & $43(81)$ \\
\hline Prosthetic valve * & $22(42)$ \\
\hline Biological & $15(28)$ \\
\hline Aortic & $11(21)$ \\
\hline Mitral & $5(10)$ \\
\hline Pulmonary & $2(4)$ \\
\hline Mechanic & $9(17)$ \\
\hline Aortic & $4(8)$ \\
\hline Mitral & $6(11)$ \\
\hline CEID & $24(45)$ \\
\hline LVAD & $4(8)$ \\
\hline Others $* *$ & $4(8)$ \\
\hline \multicolumn{2}{|l|}{ Causative pathogen, $n(\%)$} \\
\hline Positive blood culture for IE & $12(23)$ \\
\hline Coagulase Negative Staphylococcus & $3(25)$ \\
\hline Staphylococcus aureus & $4(33)$ \\
\hline Streptococcus spp. & $2(17)$ \\
\hline Enterococcus spp. & $3(25)$ \\
\hline Ongoing antibiotic treatment, $n(\%)$ & $40(75)$ \\
\hline \multicolumn{2}{|l|}{ Modified Duke Criteria, $n(\%)$} \\
\hline Duke 0 (Rejected IE) & $20(38)$ \\
\hline Duke 1 (Possible IE) & $33(62)$ \\
\hline \multicolumn{2}{|l|}{ Clinical suspicion, $n(\%)$} \\
\hline Low & $19(36)$ \\
\hline High & $34(64)$ \\
\hline \multicolumn{2}{|l|}{ IE diagnostic probability, $n(\%)$} \\
\hline Duke $0 /$ Low suspicion & $12(23)$ \\
\hline Duke $1 /$ High suspicion & $26(49)$ \\
\hline Duke 1 /Low suspicion & $7(13)$ \\
\hline Duke $0 /$ High suspicion & $8(15)$ \\
\hline Extra-cardiac FDG PET/CT infected site, $n(\%)$ & $26(49)$ \\
\hline
\end{tabular}

\subsection{Modified Duke Criteria (mDC), Clinical Suspicion Degree, and Combined $m D C /$ Clinical Suspicion}

To assess the diagnostic performances of $\mathrm{mDC}$, Duke 1 patients were considered to have a positive IE diagnosis and Duke 0 a negative IE diagnosis. In the same manner, patients with high and low suspicion were considered to have a positive and negative IE diagnosis, respectively. Combining both $\mathrm{mDC}$ and clinical suspicion degree, positive diagnosis of IE was retained for patients presenting with Duke 1/high suspicion, Duke $1 /$ low suspicion, and Duke 0/high suspicion [4]. On the other hand, patients with Duke $0 /$ low suspicion were considered to have a negative IE diagnosis [4]. Thus, according to the final diagnosis assessed by the institutional multidisciplinary Endocarditis Team, the Se, Sp, PPV, NPV, and Ac were, respectively, $84 \%, 50 \%, 48 \%, 85 \%$, and $62 \%$ for $\mathrm{mDC}$ and $95 \%, 53 \%, 53 \%, 95 \%$, and $68 \%$ for clinical suspicion degree. Taking into account both 
$\mathrm{mDC}$ and the clinical suspicion degree, the Se, Sp, PPV, NPV, and Ac were 95\%, 32\%, 44\%, $92 \%$, and $55 \%$, respectively (Table 2 ). A head-to-head comparison of global accuracy for IE diagnosis between $\mathrm{mDC}$, clinical suspicion degree, and the combination of both showed no statistically significant difference (Table 3).

Table 2. Overall diagnostic results compared to the final diagnosis, according to the Endocarditis Team consensus.

\begin{tabular}{|c|c|c|c|c|c|}
\hline & Se & $\mathrm{Sp}$ & PPV & NPV & Ac \\
\hline $\begin{array}{l}\text { Modified Duke } \\
\text { Criteria (mDC) }\end{array}$ & $84 \%(16 / 19)$ & $50 \%(17 / 34)$ & $48 \%(16 / 33)$ & $85 \%(17 / 20)$ & $62 \%(33 / 53)$ \\
\hline Degree of Clinical Suspicion & $95 \%(18 / 19)$ & $53 \%(18 / 34)$ & $53 \%(18 / 34)$ & $95 \%(18 / 19)$ & $68 \%(36 / 53)$ \\
\hline mDC+Clinical Suspicion & $95 \%(18 / 19)$ & $32 \%(11 / 34)$ & $44 \%(18 / 41)$ & $92 \%(11 / 12)$ & $55 \%(29 / 53)$ \\
\hline${ }^{18} \mathrm{~F}-\mathrm{FDG}$ PET/CT & $79 \%(15 / 19)$ & $100 \%(34 / 34)$ & $100 \%(15 / 15)$ & $89 \%(34 / 38)$ & $92 \%(49 / 53)$ \\
\hline${ }^{18}$ F-FDG PET/CT * & $83 \%(15 / 18)$ & $100 \%(25 / 25)$ & $100 \%(15 / 15)$ & $89 \%(25 / 28)$ & $93 \%(40 / 43)$ \\
\hline
\end{tabular}

Table 3. Head-to-head comparison (global accuracy for IE diagnosis) between $\mathrm{mDC}$, clinical suspicion degree, the combination of both, and ${ }^{18}$ F-FDG PET/CT.

\begin{tabular}{ccccc}
\hline & $\begin{array}{c}\text { Duke Modified } \\
\text { Criteria (mDC) }\end{array}$ & $\begin{array}{c}\text { Clinical } \\
\text { Suspicion }\end{array}$ & $\begin{array}{c}\text { mDC+Clinical } \\
\text { SUSPICION Degree }\end{array}$ & $\begin{array}{c}\text { 18F-FDG } \\
\text { PET/CT }\end{array}$ \\
\hline $\begin{array}{c}\text { Modified Duke } \\
\text { Criteria (mDC) } \\
\text { Clinical }\end{array}$ & - & $\mathrm{ns}$ & $\mathrm{ns}$ & $p=0.003$ \\
$\begin{array}{c}\text { Suspicion degree } \\
\text { mDC+Clinical }\end{array}$ & $\mathrm{ns}$ & - & $\mathrm{ns}$ & $p=0.001$ \\
$\begin{array}{l}\text { Suspicion degree } \\
\text { 18F-FDG PET/CT }\end{array}$ & $\mathrm{ns}$ & $\mathrm{ns}$ & - & $p=0.001$ \\
\hline
\end{tabular}

Using mDC, 3/20 (15\%) Duke 0 patients had a final diagnosis of IE, while the Endocarditis Team excluded IE diagnosis in 17 out of 33 (52\%) Duke 1 patients. According to clinical suspicion degree, 1 out of 19 (5\%) low suspicion patients had a final diagnosis of IE. On the other hand, in 16 out of 34 (47\%) high suspicion patients, IE diagnosis was finally excluded. Merging $\mathrm{mDC}$ and clinical suspicion degree, the diagnosis of IE was retained in 17 out of $26(65 \%)$ Duke 1/high suspicion patients, 2 out of $8(25 \%)$ Duke $0 /$ high suspicion patients, and 1 out of $12(8 \%)$ Duke $0 /$ low suspicion patients. Finally, the Endocarditis Team concluded an absence of IE in 7 out of 7 Duke 1/low suspicion patients.

\section{3. ${ }^{18} \mathrm{~F}-F D G$ PET/CT}

\subsubsection{Cardiac Area Investigation}

Forty $(75 \%)$ patients received wide spectrum antibiotic treatment at the time of the 18F-FDG PET/CT examination. In a per patient analysis (PET/CT positive or negative for IE on cardiac area), two interpreting nuclear medicine physicians were concordant for 48 of the studied 53 patients. A third physician was required for reaching a consensus in the remaining 5 patients. In the whole population, 18F-FDG PET/CT Se, Sp, PPV, NPV, and Ac for IE diagnosis were 79\%, 100\%, 100\%, 89\%, and 92\%, respectively. Considering only the 43 patients with prosthetic valves or any other cardiac devices, 18F-FDG PET/CT sensitivity was slightly better $(83 \%)$ than that reported from the analysis of the whole population (Table 2). 18F-FDG PET /CT was considered a true positive for the presence of IE in 15 out of 19 patients with final diagnosis of IE (Figures 2 and 3). 

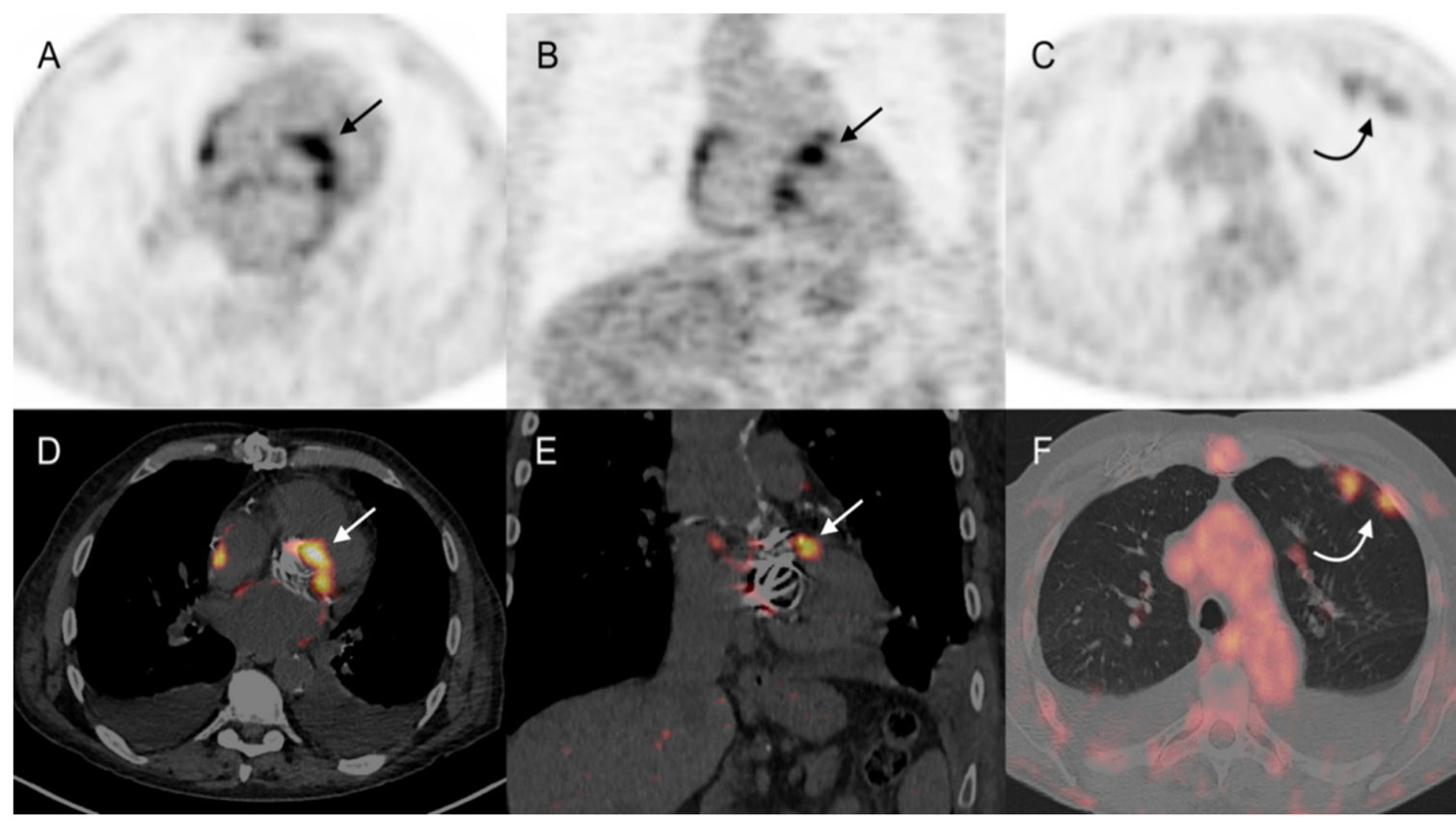

Figure 2. 73-year-old man with aortic and mitral mechanic valve prostheses presented with fever and blood cultures positive for Streptococcus gallolyticus (Duke 1/high clinical suspicion degree). TTE and TEE were both negative. PET/CT showed increased focal ${ }^{18}$ F-FDG uptake between the aortic and mitral mechanic valves (arrows) and in left lung parenchymal condensations (curved arrows). According to the Endocarditis Team, final diagnosis was an infected mechanic aortic valve with pulmonary septic emboli. (A-C): attenuation-corrected PET, axial, and coronal slices; (D-F): attenuation-corrected $\mathrm{PET} / \mathrm{CT}$, axial, and coronal slices.
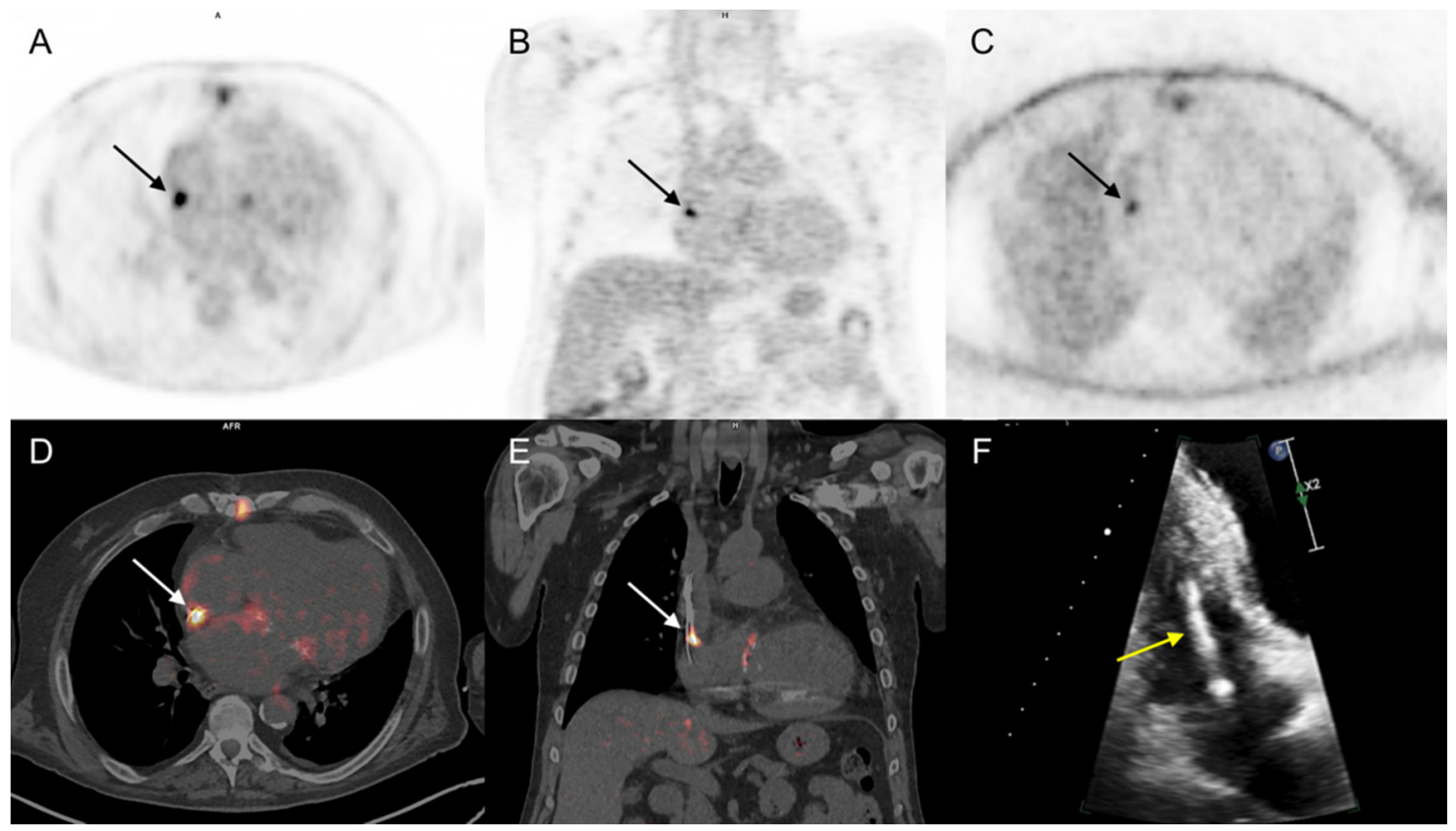

Figure 3. 68-year-old man with a history of implantable cardioverter defibrillator (ICD) and a biological prosthetic mitral valve presented with 10-mm enlargement of ICD lead within the right auricle at TTE (yellow arrow) but normal routine biological evaluation and negative blood cultures (Duke 1/high clinical suspicion degree). PET/CT showed increased focal ${ }^{18}$ F-FDG uptake, corresponding to right auricle ICD lead (arrows). The Endocarditis Team's final diagnosis was CIED IE, and ICD was removed. (A,B): PET, attenuation-corrected axial, and coronal slices. (C): PET, non-attenuation corrected axial slice. (D,E): PET/CT, axial, and coronal attenuation corrected slices. (F): TEE. 
In the remaining four patients, 18F-FDG PET/CT failed to detect the native mitral valve IE $(n=2)$, prosthetic mitral valve IE $(n=1)$, and CEID lead IE $(n=1)$. False negative results were mainly due to an insufficient decrease of physiological 18F-FDG myocardial uptake following the high fat diet (17-19). Moreover, all four patients with false negative results were treated by large spectrum antibiotics at the time of PET/CT. No false positive ${ }^{18}$ F-FDG PET/CT study was registered.

Patients with a final diagnosis of IE showed higher SUVmax values assessed on cardiac area abnormalities than no-IE patients (SUVmax: 6.2 vs. 4.9, $p=0.05$ ). However, the small sample analyzed did not allow the definition of a SUVmax diagnostic threshold for a reliable clinical use. Overall, 18F-FDG PET/CT performed significantly better than $\operatorname{mDC}(p=0.003)$, clinical suspicion degree $(p=0.001)$, and the combination of both $(p=$ 0.001) for IE diagnosis (Table 3). When 41 patients with possible or rejected IE but high clinical suspicion were analyzed jointly (4), 18F-FDG PET/CT revealed a Se, Sp, PPV, NPV, and Ac of, respectively, $78 \%, 100 \%, 100 \%, 85 \%$, and $90 \%$.

In the subgroup of 15 patients with the strongest classification discordances, including only Duke $0 /$ high suspicion $(n=8)$ and Duke $1 /$ low suspicion $(n=7),{ }^{18}$ F FDG PET $/ \mathrm{CT}$ correctly identified all patients (Table 4 ). When the results of ${ }^{18}$ F-FDG PET/CT were considered as a major criterion within ESC2015 guidelines, 1 out of 12 (8\%) rejected IE diagnoses (Duke 0/low suspicion) was changed to a possible IE diagnosis, and 11 out of 41 (27\%) possible/rejected IE diagnoses with high clinical suspicion were changed to definite IE diagnoses.

Table 4. Fifteen patients with the strongest classification discordances, including Duke $0 /$ high suspicion $(n=8)$ and Duke $1 /$ low suspicion $(n=7)$. mDc: modified Duke Criteria; IE: infective endocarditis; Rej: mDC rejected IE; Poss: mDC possible IE; PM: pace-maker; LVAD: left ventricular assistance device; TTE: transthoracic echography; TEE: transesophagus echography; Ao veg: aortic valve vegetation; Mit veg: Mitral valve vegetation; Mit abs: mitral abscess.

\begin{tabular}{|c|c|c|c|c|c|c|c|}
\hline$n^{\circ}$, Age, Sex & $\begin{array}{l}\text { mDC/Clinical } \\
\text { Suspicion }\end{array}$ & $\begin{array}{c}\text { Final } \\
\text { Diagnosis }\end{array}$ & $\begin{array}{l}\text { FDG } \\
\text { PET }\end{array}$ & TTE & TEE & $\begin{array}{c}\text { mDC Major } \\
\text { Microbiological Evidence }\end{array}$ & mDC Minor Findings \\
\hline $1,68, \mathrm{~F}$ & Rej/High & no IE & - & - & - & - & $\begin{array}{c}\text { Predisposition, microbiologic } \\
\text { evidence (Pseudomonas aeruginosa) }\end{array}$ \\
\hline $2,43, \mathrm{M}$ & Rej/High & no IE & - & - & - & - & vascular, phenomena \\
\hline $3,78, \mathrm{M}$ & Rej/High & no IE & - & - & - & - & predisposition, fever \\
\hline $4,48, \mathrm{~F}$ & Rej/High & IE & PM lead & - & - & - & Predisposition, fever \\
\hline $5,50, \mathrm{M}$ & Rej/High & IE & LVAD & - & - & - & $\begin{array}{l}\text { Fever, microbiologic evidence } \\
\text { (Staphylococcus aureus) }\end{array}$ \\
\hline $6,72, \mathrm{M}$ & Rej/High & no IE & - & - & - & - & fever \\
\hline $7,76, \mathrm{~F}$ & Rej/High & no IE & - & - & - & Staphylococcus epidermidis & - \\
\hline $8,56, \mathrm{M}$ & Rej/High & no IE & - & - & - & $\begin{array}{c}\text { Coxiella burnetii } \\
\text { IgG antiphase } 1>1 / 800\end{array}$ & - \\
\hline $9,79, \mathrm{~F}$ & Poss/Low & no IE & - & - & - & Staphylococcus aureus & fever \\
\hline $10,82, \mathrm{M}$ & Poss/Low & no IE & - & - & - & Staphylococcus epidermidis & fever \\
\hline $11,53, \mathrm{M}$ & Poss/Low & no IE & - & Ao veg & Ao veg & - & $\begin{array}{l}\text { predisposition } \\
\text { Predisposition, fever, }\end{array}$ \\
\hline $12,31, \mathrm{~F}$ & Poss/Low & no IE & - & - & - & - & $\begin{array}{l}\text { microbiologic evidence } \\
\quad \text { (Pantoea ananatis) }\end{array}$ \\
\hline $13,75, \mathrm{~F}$ & Poss/Low & no IE & - & Mit veg & Mit veg & - & fever \\
\hline $14,81, \mathrm{M}$ & Poss/Low & no IE & - & - & Mit abs & - & fever \\
\hline $15,67, \mathrm{M}$ & Poss/Low & no IE & - & - & - & Staphylococcus epidermidis & Predisposition, fever \\
\hline
\end{tabular}

\subsubsection{Extra-Cardiac Infection Assessment}

In 9 out of 19 patients with a final diagnosis of IE, PET/CT showed extra-cardiac 18F-FDG pathologic uptake in: (a) lung $(n=3)$ and bone $(n=1)$, suggesting embolic spread (Figure 2); (b) previously documented spondylodiscitis $(n=1)$ and infected hip and knee prosthesis $(n=2)$, which were afterwards identified as the source of IE; (c) parietal collections $(n=2)$ corresponding to post-thoracotomy abscesses, which were finally considered as concomitant infections independent from IE. Concerning the 34 patients with excluded IE diagnosis, 18F-FDG PET/CT showed pathologic extra-cardiac uptake in 17 cases, strongly suggesting lung infections $(n=6)$, spondylodiscitis $(n=3)$, postthoracotomy local abscess $(n=1)$, infected knee prosthesis $(n=1)$, infected central catheters $(n=2)$, colitis $(n=1)$, infected deep venous thrombosis $(n=1)$, LVAD cable infection in the abdominal path $(n=1)$, and infected acute pancreatitis associated with cholecystitis 
$(n=1)$ when correlated with patient follow up. In 6 out of 12 patients with initially rejected (Duke 0/Low suspicion) and finally excluded IE diagnoses, 18F-FDG PET/CT contributed to detected lung infections $(n=3)$, infected acute pancreatitis associated with cholecystitis $(n=1)$, infected central catheter $(n=1)$, and infection of the extra thoracic cable of a LVAD $(n=1)$.

\subsubsection{Impact on Patient Management}

The real therapeutic impact of 18F-FDG PET/CT in a retrospective setting could be difficult to assess. Patient therapeutic strategy was led by the Endocarditis team. Accordingly, 18F-FDG PET/CT contributed to patient management in 24 out of 53 (45\%) cases. In particular, PET/CT was considered contributory when it fulfilled at least one of the following criteria: (a) identification of the presence of cardiac infection (other conventional tests performed at the time of PET/CT imaging were negative and/or non-contributory) ( $n=15,28 \%)$, (b) determination of the extent of the infection or the involvement of other organs $(n=4,8 \%)$, (c) removal of the infection site $(n=3,6 \%$, including 2 CEID removals and 1 PV replacement), and (d) change in the nature and duration of antibiotic therapy $(n=14,26 \%)$ : beginning of specific antibiotic treatment $(n=2)$ and modification of antibiotic spectrum $(n=12)$.

\section{Discussion}

The main findings that can be drawn from our retrospective study are in line with ESC2015 recommendations, highlighting the good performance of ${ }^{18} \mathrm{~F}-\mathrm{FDG}$ PET/CT for the diagnosis of IE, doing better than the $\mathrm{MDC}$, the degree of clinical suspicion, and the combination of both [17-23]. ${ }^{18}$ F-FDG PET/CT sensitivity for IE detection was higher in patients with cardiac devices than in the overall population ( $83 \%$ vs. $79 \%$, respectively). Moreover, ${ }^{18} \mathrm{~F}-\mathrm{FDG}$ PET/CT was efficient for 41 patients with possible/rejected IE but high clinical suspicion (Se: 78\%), a situation which seems to represent the most difficult cases [4] Finally, ${ }^{18}$ F-FDG PET /CT correctly characterized all 15 patients classified as Duke $0 /$ high suspicion and Duke $1 /$ low suspicion.

The clinical interest of ${ }^{18} \mathrm{~F}-\mathrm{FDG}$ PET/CT in patients with possible/rejected IE but high clinical suspicion or rejected IE and low clinical suspicion needs further evaluation. Therefore, we have challenged ${ }^{18} \mathrm{~F}$-FDG PET/CT by including only patients without a definite diagnosis of IE, according to $\mathrm{mDC}$ (i.e., only Duke 0 and 1). Consequently, patients presented with elevated inflammatory blood markers or at least one minor criterion according to $\mathrm{mDC}$ (Duke 0 and low clinical suspicion) were included in the analysis. ${ }^{18} \mathrm{~F}-\mathrm{FDG}$ PET/CT for these patients were largely performed before publication of the ESC2015 guidelines. Finally, a sensitivity of $78 \%$ in patients with possible or rejected IE but high clinical suspicion appears robust. Moreover, ${ }^{18}$ F-FDG PET/CT plays a role in patient management in 24 out of $53(45 \%)$ cases, in accordance with previously reported data $[9,10,24]$.

Despite disagreements in the literature, there is a growing interest for early ${ }^{18} \mathrm{~F}$-FDG PET/CT use in the diagnostic algorithm in IE suspicion [10,11]. Moreover, early ${ }^{18} \mathrm{~F}-\mathrm{FDG}$ PET/CT for IE suspicion seems to lead to a prompt treatment and a better prognosis [25]. The inclusion of ${ }^{18} \mathrm{~F}$-FDG PET/CT in the ESC2015 guidelines led to a 3-scale degree of IE probability, resulting in numerous doubtful diagnostic situations. In our study, when ${ }^{18}$ F-FDG PET/CT was considered as a major criterion for IE diagnosis in the ESC2015 guidelines, 12 and 11 patients were respectively reclassified from possible into definite and rejected IE. However, the diagnosis of possible IE persisted as unchanged for the remaining 30 patients $(57 \%)$. According to our findings, ${ }^{18} \mathrm{~F}-\mathrm{FDG}$ PET/CT defines the diagnosis as either rejected or definite IE. Thus, adding ${ }^{18} \mathrm{~F}$-FDG PET/CT directly to $\mathrm{mDC}$ at patient admission could promptly reduce the number of possible and rejected IE by increasing specificity, without a major change of sensitivity.

In our series, ${ }^{18}$ F-FDG PET/CT failed to detect native mitral valve IE in two patients. According to recent data, PET cardiac studies should be performed as ECG-gated and 
contrast-enhanced PET/CTA to increase the diagnostic accuracy, particularly for the evaluation of native valves [16,26-28]. However, both techniques are not widely available in all nuclear medicine laboratories, and the present study could be considered as an illustration of "real life" ${ }^{18}$ F-FDG PET/CT utilization. In our series, ${ }^{18}$ F-FDG PET/CT showed optimal specificity without achieving false positive results and good diagnostic accuracy, despite the absence of ECG-gated PET acquisition and contrast media injection. These findings are the consequences of an expert qualitative analysis using well-defined interpretation criteria, as previously mentioned [12,13,29].

CIED represents a specific diagnostic and therapeutic challenge. In our study, 18F-FDG PET/CT failed to detect one patient with CEID-lead IE. Intra-cardiac echocardiography (ICE) is a diagnostic alternative, without patient radiation exposure, useful in high clinical suspicion of CIED when TTE and TEE are non-conclusive. However, it rests on an invasive diagnostic technique [30,31].

Semi-quantitative analysis using standardized uptake (SUV) has been proposed but not validated in inflammation and infection. Several authors previously reported conflicting results without reaching a definitive threshold that would allow to distinguish between possible and rejected IE diagnosis in daily clinical practices [14,15]. The additional value of quantitative parameters, such as SUVmax, in differentiating between infected and noninfected material is still debated. New semiquantitative indices have recently been examined but need further confirmation [32].

Four patients with a final diagnosis of IE reported a false negative result from their PET/CT investigation. All of these patients were being treated by large spectrum antibiotics at the time of the PET/CT examination. Chronic or indolent infections with slow-growing bacteria, or infections with bacteria in a quiescent status after long-term antibiotic treatments, are common causes of false negative ${ }^{18}$ F-FDG PET/CT results. Moreover, in clinical practice, most patients with suspicion of IE generally have started antibiotic therapy before the PET/CT examination. Therefore, it is advisable to conduct a ${ }^{18} \mathrm{~F}-\mathrm{FDG}$ PET/CT study before treatment initiation or as quickly as possible to avoid potential underestimation of disease extent and severity. If there is clinical suspicion of infection, a negative result authorizes clinical observation without immediate surgery, while a positive ${ }^{18}$ F-FDG PET/CT finding in the clinical context may lead to the extraction of the entire device. Thus, it is important to bear in mind that a false-negative PET result, which is often related to a mild or chronic infection after or during the antibiotic treatment, may not really influence conservative management and has a low risk of exposing the patient to clinical deterioration.

Recent studies show that early identification of embolic events or infection sources using ${ }^{18}$ F-FDG PET/CT means early antimicrobial therapy (adapted to a suspected causing pathogen according to the infectious gateway), prolonged or modified treatment, or even early surgical cardiac valve procedures $[33,34]$. In our study, ${ }^{18}$ F-FDG PET/CT suggested extra-cardiac infectious processes in 6 out of 12 patients with a final excluded IE. Concerning the 19 patients with a confirmed IE, ${ }^{18}$ F-FDG PET/CT revealed extra-cardiac pathological uptake in 9 out of 19 (47\%) of them, which was determined to be related to either septic embolic events, sources of the infection, or concomitant infection. These data match the performances of ${ }^{18} \mathrm{~F}-\mathrm{FDG} \mathrm{PET} / \mathrm{CT}$ in the assessment of unexplained chronic fever or inflammatory syndrome [35].

The use of multimodality imaging could be of great interest for the management of IE patients [36]. Functional techniques will be variably associated with morphological investigations. A typical example of diagnostic synergy is the combined use of ${ }^{18}$ F-FDG PET and CTA in a single PET/CTA examination [15]. Nowadays, PET/MRI hybrid systems decrease the effective radiation doses and are beginning to be clinically available. However, further studies with long-term economic considerations will be needed to validate and support the necessity for this imaging approach. The optimal combination of imaging techniques necessitates determination to improve the diagnostic accuracy and reduce non-essential patient radiation exposure $[37,38]$. 
This study presents several limitations that deserve comments: first, its retrospective and monocentric design focused on a heterogeneous population, including cases of PVE, devices, and LVAD infection. However, it could be considered as a basis for future investigational prospective multicenter studies, including a larger number of patients with challenging endocarditis on native valves and devices, proving that ${ }^{18} \mathrm{~F}$-FDG PET/CT is feasible in most centers. Second, the number of included patients is also quite small compared to recent studies on the same topic, which is mainly related to different inclusion criteria. Indeed, in our work, patients with a definite diagnosis of IE were not included, contrarily to other recent studies based on larger patient cohorts. Next, the clinical suspicion degree of IE diagnosis was established by the Infectious Disease Specialist Consensus, which could be based on relatively subjective criteria but does represent a picture of real-life clinical practices. Finally, as previously mentioned, some patients had ${ }^{18} \mathrm{~F}-\mathrm{FDG}$ PET/CT exploration before publication of the ESC guidelines, potentially representing a patient selection bias.

\section{Conclusions}

${ }^{18} \mathrm{~F}$-FDG PET/CT represents a valuable diagnostic tool that should be considered in patients with IE suspicion, even for those challenging cases with significant inconsistencies between $\mathrm{mDC}$ and clinical IE presumption. In this respect, ${ }^{18} \mathrm{~F}-\mathrm{FDG}$ PET/CT may afford a binary diagnosis (definite or rejected IE) by removing uncertain diagnostic situations, thus improving patient therapeutic management.

Author Contributions: Conceptualization, V.P., C.B., Y.R., M.M., S.E.G., O.M., Y.H., T.H.S., and A.I.; methodology, V.P., C.B., Y.R., M.M., S.E.G., O.M., Y.H., T.H.S., and A.I.; validation, V.P., C.B., Y.R., M.M., S.E.G., O.M., Y.H., T.H.S., and A.I.; formal analysis, V.P., C.B., Y.R., M.M., S.E.G., O.M., Y.H., T.H.S., and A.I.; data curation, V.P., C.B., Y.R., M.M., S.E.G., O.M., Y.H., T.H.S., and A.I.; writingoriginal draft preparation, V.P.; writing-review and editing, C.B. and A.I.; supervision, A.I. All authors have read and agreed to the published version of the manuscript.

Funding: This research received no external funding.

Institutional Review Board Statement: The study was conducted according to the guidelines of the Declaration of Helsinki. Retrospective non-interventional studies do not require IRB/IEC in France.

Informed Consent Statement: Informed written consent was obtained from all subjects involved in the study.

Data Availability Statement: Purely observational studies do not require registration.

Conflicts of Interest: The authors declare no conflict of interest.

\section{References}

1. Thuny, F.; Grisoli, D.; Collart, F.; Habib, G.; Raoult, D. Management of infective endocarditis: Challenges and perspectives. Lancet 2012, 379, 965-975. [CrossRef]

2. Slipczuk, L.; Codolosa, J.N.; Davila, C.D.; Romero-Corral, A.; Yun, J.; Pressman, G.S.; Figueredo, V.M. Infective Endocarditis Epidemiology Over Five Decades: A Systematic Review. PLoS ONE 2013, 8, e82665. [CrossRef] [PubMed]

3. Li, J.S.; Sexton, D.J.; Mick, N.; Nettles, R.; Fowler, J.V.G.; Ryan, T.; Bashore, T.; Corey, G.R. Proposed Modifications to the Duke Criteria for the Diagnosis of Infective Endocarditis. Clin. Infect. Dis. 2000, 30, 633-638. [CrossRef]

4. Habib, G.; Lancellotti, P.; Antunes, M.J.; Bongiorni, M.G.; Casalta, J.-P.; Del Zotti, F.; Dulgheru, R.; El Khoury, G.; Erba, P.A.; Iung, B.; et al. 2015 ESC Guidelines for the management of infective endocarditis. The Task Force for the Management of Infective Endocarditis of the European Society of Cardiology (ESC). Eur. Heart J. 2015, 36, 3075-3128. [CrossRef]

5. Vieira, M.L.C.; Grinberg, M.; Pomerantzeff, P.M.A.; Andrade, J.L.; Mansur, A.J. Repeated echocardiographic examinations of patients with suspected infective endocarditis. Heart 2004, 90, 1020-1024. [CrossRef]

6. Bruun, N.E.; Habib, G.; Thuny, F.; Sogaard, P. Cardiac imaging in infectious endocarditis. Eur. Heart J. 2014, 35, 624-632. [CrossRef]

7. Rouzet, F.; Chequer, R.; Benali, K.; Lepage, L.; Ghodbane, W.; Duval, X.; Iung, B.; Vahanian, A.; Le Guludec, D.; Hyafil, F. Respective Performance of 18F-FDG PET and Radiolabeled Leukocyte Scintigraphy for the Diagnosis of Prosthetic Valve Endocarditis. J. Nucl. Med. 2014, 55, 1980-1985. [CrossRef] 
8. Sánchez-Enrique, C.; Olmos, C.; Jiménez-Ballvé, A.; Fernández-Pérez, C.; Ferrera, C.; Pérez-Castejón, M.J.; Candil, A.O.; DelgadoBolton, R.; Carnero, M.; Maroto, L.; et al. Usefulness of 18F Fluorodeoxyglucose Positron Emission Tomography/Computed Tomography in Infective Endocarditis in Daily Practice. JACC Cardiovasc. Imaging 2018, 11, 1920-1922. [CrossRef]

9. Chen, W.; Sajadi, M.M.; Dilsizian, V. Merits of FDG PET/CT and Functional Molecular Imaging Over Anatomic Imag-ing With Echocardiography and CT Angiography for the Diagnosis of Cardiac Device Infections. JACC Cardiovasc. Imaging 2018, 11, 1679-1691. [CrossRef]

10. Duval, X.; Le Moing, V.; Tubiana, S.; Esposito-Farèse, M.; Ilic-Habensus, E.; Leclercq, F.; Bourdon, A.; Goehringer, F.; Selton-Suty, C.; Chevalier, E.; et al. Impact of Systematic Whole-body 18F-Fluorodeoxyglucose PET/CT on the Management of Patients Suspected of Infective Endocarditis: The Prospective Multicenter TEPvENDO Study. Clin. Infect. Dis. 2020. [CrossRef] [PubMed]

11. Philip, M.; Tessonier, L.; Mancini, J.; Mainardi, J.-L.; Fernandez-Gerlinger, M.-P.; Lussato, D.; Attias, D.; Cammilleri, S.; Weinmann, P.; Hagege, A.; et al. Comparison Between ESC and Duke Criteria for the Diagnosis of Prosthetic Valve Infective Endocarditis. JACC Cardiovasc. Imaging 2020, 13, 2605-2615. [CrossRef]

12. Mathieu, C.; Mikaïl, N.; Benali, K.; Iung, B.; Duval, X.; Nataf, P.; Jondeau, G.; Hyafil, F.; Le Guludec, D.; Rouzet, F. Characterization of 18F-Fluorodeoxyglucose Uptake Pattern in Noninfected Prosthetic Heart Valves. Circ. Cardiovasc. Imaging. 2017, 10, e005585. [PubMed]

13. Scholtens, A.M.; Swart, L.E.; Verberne, H.J.; Tanis, W.; Lam, M.G.; Budde, R.P. Confounders in FDG-PET/CT Imaging of Suspected Prosthetic Valve Endocarditis. JACC Cardiovasc. Imaging 2016, 9, 1462-1465. [CrossRef]

14. Calais, J.; Touati, A.; Grall, N.; Laouénan, C.; Benali, K.; Mahida, B.; Vigne, J.; Hyafil, F.; Iung, B.; Duval, X.; et al. Diagnostic Impact of 18 F-Fluorodeoxyglucose Positron Emission Tomography/Computed Tomography and White Blood Cell SPECT/Computed Tomography in Patients With Suspected Cardiac Implantable Electronic Device Chronic Infection. Circ. Cardiovasc. Imaging 2019, 12, e007188. [CrossRef] [PubMed]

15. Pizzi, M.N.; Roque, A.; Fernández-Hidalgo, N.; Cuéllar-Calabria, H.; Ferreira-González, I.; Gonzàlez-Alujas, M.T.; Oristrell, G.; Gracia-Sánchez, L.; González, J.J.; Rodríguez-Palomares, J.; et al. Improving the Diagnosis of Infective Endocarditis in Prosthetic Valves and Intracardiac Devices With 18F-Fluordeoxyglucose Positron Emission Tomography/Computed Tomography Angiography: Initial Results at an Infective Endocarditis Referral Center. Circulation 2015, 132, 1113-1126. [CrossRef] [PubMed]

16. Erba, P.A.; Lancellotti, P.; Vilacosta, I.; Gaemperli, O.; Rouzet, F.; Hacker, M.; Signore, A.; Slart, R.H.J.A.; Habib, G. Recommendations on nuclear and multimodality imaging in IE and CIED infections. Eur. J. Nucl. Med. Mol. Imaging 2018, 45, 1795-1815. [CrossRef] [PubMed]

17. Gomes, A.; Glaudemans, A.W.J.M.; Touw, D.J.; van Melle, J.P.; Willems, T.P.; Maass, A.H.; Natour, E.; Prakken, N.H.J.; Borra, R.J.H.; van Geel, P.P.; et al. Diagnostic value of imaging in infective endocarditis: A systematic review. Lancet Infect. Dis. 2017, 17, e1-e14. [CrossRef]

18. Swart, L.E.; Gomes, A.; Scholtens, A.M.; Sinha, B.; Tanis, W.; Lam, M.G.; van der Vlugt, M.J.; Streukens, S.A.F.; Aarntzen, E.H.; Bucerius, J.; et al. Improving the Diagnostic Performance of 18 F-Fluorodeoxyglucose Positron-Emission Tomography/Computed Tomography in Prosthetic Heart Valve Endocarditis. Circulation 2018, 138, 1412-1427. [CrossRef] [PubMed]

19. Scholtens, A.; Van Aarnhem, E.; Budde, R. Effect of antibiotics on FDG-PET/CT imaging of prosthetic heart valve endocarditis. Eur. Heart J. Cardiovasc. Imaging 2015, 16, 1223. [CrossRef] [PubMed]

20. Sag, S.J.M.; Menhart, K.; Grosse, J.; Hitzenbichler, F.; Hanses, F.; Mohr, A.; Salzberger, B.; Zerdzitzki, M.; Hilker, M.; Rupprecht, L.; et al. Diagnostic value of FDG PET/CT imaging in patients with surgically managed infective endocarditis: Results of a retrospective analysis at a tertiary center. J. Nucl. Cardiol. 2020, 22, 1-14. [CrossRef]

21. Abikhzer, G.; Martineau, P.; Grégoire, J.; Finnerty, V.; Harel, F.; Pelletier-Galarneau, M. [18F]FDG-PET CT for the evaluation of native valve endocarditis. J. Nucl. Cardiol. 2020, 16, 1-8. [CrossRef] [PubMed]

22. El-Dalati, S.; Murthy, V.L.; Owczarczyk, A.B.; Fagan, C.; Riddell, J., 4th; Cinti, S.; Weinberg, R.L. Correlating cardiac F-18 FDG $\mathrm{PET} / \mathrm{CT}$ results with intra-operative findings in infectious endocarditis. J. Nucl Cardiol. 2021, 28, 289-294. [CrossRef]

23. Chen, W.; Dilsizian, V. FDG PET/CT for the diagnosis and management of infective endocarditis: Expert consensus vs evidencebased practice. J. Nucl. Cardiol. 2018, 26, 313-315. [CrossRef] [PubMed]

24. Lakkas, L.; Serim, B.D.; Fotopoulos, A.; Iakovou, I.; Doumas, A.; Korkmaz, U.; Michalis, L.K.; Sioka, C. Infection of cardiac prosthetic valves and implantable electronic devices: Early diagnosis and treatment. Acta Cardiol. 2020, 14, 1-7. [CrossRef] [PubMed]

25. De Camargo, R.A.; Sommer Bitencourt, M.; Meneghetti, J.C.; Soares, J., Jr.; Gonçalves, L.F.T.; Buchpiguel, C.A.; Paixão, M.R.; Felicio, M.F.; de Matos Soeiro, A.; Strabelli, T.M.V.; et al. The Role of 18F-Fluorodeoxyglucose Positron Emission Tomography/Computed Tomography in the Diagnosis of Left-sided Endocarditis: Native vs Prosthetic Valves Endocarditis. Clin. Infect. Dis. Off. Publ. Infect. Dis. Soc. Am. 2020, 70, 583-594. [CrossRef] [PubMed]

26. Wahadat, A.R.; Tanis, W.; Swart, L.E.; Scholtens, A.; Krestin, G.P.; Van Mieghem, N.M.D.A.; Schurink, C.A.M.; Van Der Spoel, T.I.G.; Brink, F.S.V.D.; Vossenberg, T.; et al. Added value of 18F-FDG-PET/CT and cardiac CTA in suspected transcatheter aortic valve endocarditis. J. Nucl. Cardiol. 2019, 2, 1-11. [CrossRef]

27. Boursier, C.; Duval, X.; Bourdon, A.; Imbert, L.; Mahida, B.; Chevalier, E.; Claudin, M.; Hoen, B.; Goehringer, F.; Selton-Suty, C.; et al. ECG-Gated Cardiac FDG PET Acquisitions Significantly Improve Detectability of Infective Endocarditis. JACC Cardiovasc. Imaging 2020, 13, 2691-2693. [CrossRef] 
28. Kamani, C.H.; Allenbach, G.; Jreige, M.; Pavon, A.G.; Meyer, M.; Testart, N.; Firsova, M.; Vieira, V.F.; Boughdad, S.; LaLonde, M.N.; et al. Diagnostic Performance of ${ }^{18}$ F-FDG PET/CT in Native Valve Endocarditis: Systematic Review and Bivariate Meta-Analysis. Diagnostics 2020, 10, 754. [CrossRef]

29. Wahadat, A.R.; Tanis, W.; Scholtens, A.M.; Bekker, M.; Graven, L.H.; Swart, L.E.; den Harder, A.M.; Lam, M.G.; De Heer, L.M.; Roos-Hesselink, J.W.; et al. Normal imaging findings after aortic valve implantation on 18F-Fluorodeoxyglucose positron emission tomography with computed tomography. J. Nucl. Cardiol. Off. Publ. Am. Soc. Nucl. Cardiol. 2020. [CrossRef]

30. Caiati, C.; Pollice, P.; Lepera, M.E.; Favale, S. Pacemaker Lead Endocarditis Investigated with Intracardiac Echocardiography: Factors Modulating the Size of Vegetations and Larger Vegetation Embolic Risk during Lead Extraction. Antibiotics 2019 , 8, 228. [CrossRef]

31. Narducci, M.L.; Pelargonio, G.; Russo, E.; Marinaccio, L.; Di Monaco, A.; Perna, F.; Bencardino, G.; Casella, M.; Di Biase, L.; Santangeli, P.; et al. Usefulness of Intracardiac Echocardiography for the Diagnosis of Cardiovascular Implantable Electronic Device-Related Endocarditis. J. Am. Coll. Cardiol. 2013, 61, 1398-1405. [CrossRef]

32. Roque, A.; Pizzi, M.N.; Fernández-Hidalgo, N.; Permanyer, E.; Cuellar-Calabria, H.; Romero-Farina, G.; Ríos, R.; Almirante, B.; Castell-Conesa, J.; Escobar, M.; et al. Morpho-metabolic post-surgical patterns of non-infected prosthetic heart valves by [18F]FDG PET/CTA: "normality" is a possible diagnosis. Eur. Heart J. Cardiovasc. Imaging 2019, 21, 24-33. [CrossRef] [PubMed]

33. Holle, S.L.K.; Andersen, M.H.; Klein, C.F.; Bruun, N.E.; Tønder, N.; Haarmark, C.; Loft, A.; Høilund-Carlsen, P.F.; Bundgaard, H.; Iversen, K.K. Clinical usefulness of FDG-PET/CT for identification of abnormal extra-cardiac foci in patients with infective endocarditis. Int. J. Cardiovasc. Imaging 2020, 36, 939-946. [CrossRef]

34. Orvin, K.; Goldberg, E.; Bernstine, H.; Groshar, D.; Sagie, A.; Kornowski, R.; Bishara, J. The role of FDG-PET/CT imaging in early detection of extra-cardiac complications of infective endocarditis. Clin. Microbiol. Infect. 2015, 21, 69-76. [CrossRef]

35. Federici, L.; Blondet, C.; Imperiale, A.; Sibilia, J.; Pasquali, J.-L.; Pflumio, F.; Goichot, B.; Blaison, G.; Weber, J.-C.; Christmann, D.; et al. Value of 18F-FDG-PET/CT in patients with fever of unknown origin and unexplained prolonged inflammatory syndrome: A single centre analysis experience. Int. J. Clin. Pract. 2010, 64, 55-60. [CrossRef] [PubMed]

36. Gomes, A.; Van Geel, P.P.; Santing, M.; Prakken, N.H.J.; Ruis, M.L.; Van Assen, S.; Slart, R.H.J.A.; Sinha, B.; Glaudemans, A.W.J.M. Imaging infective endocarditis: Adherence to a diagnostic flowchart and direct comparison of imaging techniques. J. Nucl. Cardiol. 2020, 27, 592-608. [CrossRef] [PubMed]

37. Li, Y.; Jiang, L.; Wang, H.; Cai, H.; Xiang, Y.; Li, L. Effective radiation dose of 18f-fdg pet/ct: How much does diagnostic ct contribute? Radiat. Prot. Dosim. 2019, 187, 183-190. [CrossRef]

38. Nguyen, P.K.; Lee, W.H.; Li, Y.F.; Hong, W.X.; Hu, S.; Chan, C.; Liang, G.; Nguyen, I.; Ong, S.-G.; Churko, J.; et al. Assessment of the Radiation Effects of Cardiac CT Angiography Using Protein and Genetic Biomarkers. JACC Cardiovasc. Imaging 2015, 8 , 873-884. [CrossRef] 